\title{
Evaluating anesthetic protocols for functional blood flow imaging in the rat eye
}

Eric M. Moult

WooJhon Choi

David A. Boas

Bernhard Baumann

Allen C. Clermont

Edward P. Feener

James G. Fujimoto 


\title{
Evaluating anesthetic protocols for functional blood flow imaging in the rat eye
}

\author{
Eric M. Moult, ${ }^{a, \dagger}$ WooJhon Choi, ${ }^{a, \dagger}$ David A. Boas, ${ }^{b}$ Bernhard Baumann, ${ }^{a, c}$ Allen C. Clermont, ${ }^{d}$ \\ Edward P. Feener, ${ }^{\mathrm{d}}$ and James G. Fujimoto ${ }^{\mathrm{a}, *}$ \\ a Massachusetts Institute of Technology, Research Laboratory of Electronics, Department of Electrical Engineering and Computer Science, \\ 50 Vassar Street, Cambridge 02139, United States \\ bHarvard Medical School, Massachusetts General Hospital, Athinoula A. Martinos Center for Biomedical Imaging, Department of Radiology, \\ 13th Street, Charlestown 02129, United States \\ ${ }^{c}$ Medical University of Vienna, Center for Medical Physics and Biomedical Engineering, Waehringer Guertel 18, Vienna 1090, Austria \\ dJoslin Diabetes Center, Research Division, One Joslin Place, Boston 02215, United States
}

\begin{abstract}
The purpose of this study is to evaluate the suitability of five different anesthetic protocols (isoflurane, isoflurane-xylazine, pentobarbital, ketamine-xylazine, and ketamine-xylazine-vecuronium) for functional blood flow imaging in the rat eye. Total retinal blood flow was measured at a series of time points using an ultrahighspeed Doppler OCT system. Additionally, each anesthetic protocol was qualitatively evaluated according to the following criteria: (1) time-stability of blood flow, (2) overall rate of blood flow, (3) ocular immobilization, and (4) simplicity. We observed that different anesthetic protocols produced markedly different blood flows. Different anesthetic protocols also varied with respect to the four evaluated criteria. These findings suggest that the choice of anesthetic protocol should be carefully considered when designing and interpreting functional blood flow studies in the rat eye. ๑ The Authors. Published by SPIE under a Creative Commons Attribution 3.0 Unported License. Distribution or reproduction of this work in whole or in part requires full attribution of the original publication, including its DOI. [DOI: 10.1117/1.JBO.22.1.016005]
\end{abstract}

Keywords: anesthesia; functional imaging; small animal; blood flow; Doppler OCT; rat.

Paper 160637R received Sep. 14, 2016; accepted for publication Dec. 15, 2016; published online Jan. 5, 2017.

\section{Introduction}

Measurement of small animal ocular blood flow is of significant interest to the scientific and clinical research communities. This interest is attributable to the importance of ocular blood flow as a parameter in studies of ocular pathophysiology and its potential to serve as an early marker of a variety of ocular diseases, including age-related macular degeneration, diabetic retinopathy, and glaucoma. The importance of ocular blood flow has driven the development of multiple measurement technologies including retinal/dynamic vessel analysis, ${ }^{1}$ microsphere methods, ${ }^{2-4}$ laser Doppler-based velocimetry ${ }^{5-8}$ and flowmetry, ${ }^{9-11}$ laser speckle imaging, ${ }^{11-13}$ magnetic resonance imaging (MRI), ${ }^{14-16}$ color Doppler ultrasound imaging, ${ }^{17-19}$ and optical coherence tomography (OCT).$^{20-31}$ En face Doppler OCT, the modality used in this study, is a noninvasive, OCT-based technique that measures the Doppler velocities in the en face plane. In contrast to traditional Doppler OCT, which uses individual cross-sectional images, en face Doppler OCT does not require determination of the Doppler angle. Instead, in en face Doppler OCT, total blood flow is obtained by segmenting the blood vessels and integrating Doppler velocities in the en face plane. $^{26,28,32}$

Murine models are widely used to study the pathology of and develop treatments for ocular diseases. Anesthesia is a common requirement prior to small animal imaging, and it is well known within the neuroscience community that different anesthetics elicit different cerebral blood flows. ${ }^{33-35}$ However, there have been relatively few studies examining the influence of

\footnotetext{
*Address all correspondence to: James G. Fujimoto, E-mail: jgfuji@ mit.edu
}

${ }^{\dagger}$ Both authors contributed equally to this study. anesthetic protocols on ocular blood flow in the rat. Muir and Duong ${ }^{14}$ compared the effects of isoflurane and ketamine-xylazine on ocular blood flow in the mouse eye. Using arterial spin labeling (ASL) MRI they found that ketamine-xylazine anesthesia (100 mg/kg ketamine and $10 \mathrm{mg} / \mathrm{kg}$ xylazine) had associated blood flow values that were $29 \%$ lower $(0.88 \pm 0.22 \mathrm{~mL} / \mathrm{g} / \mathrm{min}$ versus $1.3 \pm 0.44 \mathrm{~mL} / \mathrm{g} / \mathrm{min})$ in the retinal tissues $(p<0.01)$ and $42 \%$ lower $(4.3 \pm 1.9 \mathrm{~mL} / \mathrm{g} / \mathrm{min}$ versus $7.7 \pm 2.1 \mathrm{~mL} / \mathrm{g} /$ $\mathrm{min})$ in the choroidal tissues $(p<0.01)$ compared to flows during isoflurane anesthesia (1.1\%). In another study using ASL MRI, Li et al. ${ }^{15}$ measured total ocular blood flow in rats anesthetized under two different isoflurane dosages. Under $1.0 \%$ isoflurane anesthesia the basal blood flow was $6.3 \pm 1.0 \mathrm{~mL} / \mathrm{g} / \mathrm{min}$, while under $1.5 \%$ isoflurane the basal blood flow was $9.3 \pm 2.7 \mathrm{~mL} / \mathrm{g} / \mathrm{min}$. At the resolution used in that study, the reported MRI blood flow measurements were a weighted average of the blood flows in the choroid and retina.

The purpose of this study is to evaluate the suitability of different anesthetic protocols for functional blood flow imaging in the rat eye; in large part, this study was motivated by the desire to develop an anesthetic protocol allowing for repeatable, controlled blood flow measurements in longitudinal studies. Using an ultrahigh-speed en face Doppler OCT system, we quantitatively measure total retinal blood flow in male Sprague-Dawley rats anesthetized with the following protocols: (1) isoflurane; (2) isoflurane-xylazine, with xylazine delivered via intraperitoneal (IP) injection; (3) pentobarbital via IP injection; (4) ketamine-xylazine via IP injection; and (5) ketamine-xylazine in conjunction with vecuronium bromide (a paralytic agent), with all three agents delivered via continuous rate infusion (CRI). In addition to providing quantitative total retinal blood flow at a series of time points, we evaluate each protocol 
according to the following criteria: (1) time-stability of blood flow, (2) overall rate of blood flow and its potential interference with functional blood flow measurements, (3) ocular immobilization, and (4) simplicity.

\section{Methods}

\subsection{Animal Preparation and Imaging Protocol}

Male Sprague-Dawley rats (Charles River Laboratories), whose numbers and weights are described in Table 1, were used. The animals were kept in the Massachusetts Institute of Technology's (MIT) Department of Comparative Medicine's veterinary housing unit and were given water and food ad libitum. All procedures were approved by MIT's Committee on Animal Care and abide with the National Institutes of Health guide for the care and use of laboratory animals.

Anesthetic dosages and routes of administration are summarized in Table 2. Dosages for ketamine-xylazine and pentobarbital, while close to surgical levels, were selected as such to minimize ocular motion. For the ketamine-xylazine-vecuronium regime, rats were anesthetized with $2 \%$ isoflurane prior to venous catheterization. Isoflurane was discontinued after the ketamine-xylazine CRI was initiated. After tracheal intubation was nonsurgically performed, ventilation was initiated followed with vecuronium CRI (in addition to ketamine-xylazine CRI). Note that immediately prior to the initiation of the ketamine-xylazine-vecuronium CRI, a bolus intravenous (IV) injection of vecuronium was administered. Finally, for all IP anesthetic protocols, so that time-transient behavior would be apparent, maintenance dosages were not delivered subsequent to the initial bolus injection.

After anesthesia, rats were placed in a custom holder that allowed them to be securely manipulated during imaging. For each rat, Doppler OCT measurements were repeatedly acquired to create a time-series of blood flow values. Prior to each Doppler OCT measurement, the OCT beam was manually aligned on the animal's eye to maximize signal strength. Throughout the procedure, the animal's heart rate and blood oxygen saturation were measured with a pulse oximeter (Nonin) and blood pressure was measured using a tail cuff (Kent Scientific). Temperature was measured using a rectal probe and was maintained with an electronically controlled thermal blanket (Harvard Apparatus). Intraocular pressure was measured using a rebound tonometer (Icare, Finland) before each Doppler OCT acquisition. A mix of medical air and oxygen was provided as needed to keep blood oxygen saturation levels above $95 \%$. For the ketaminexylazine-vecuronium protocol, rats were ventilated using the Inspira Advanced Safety Ventilator (Harvard Apparatus).
Table 2 Summary of the dosages and methods of delivery.

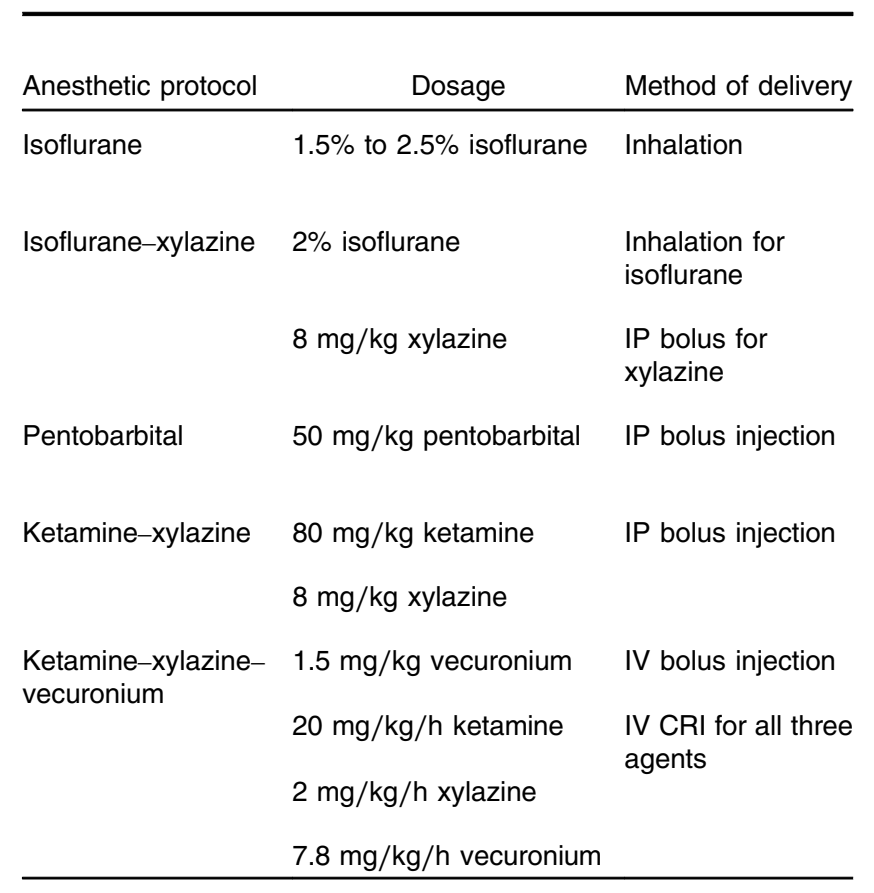

Note: CRI, continuous rate infusion; IP, intraperitoneal; IV, intravenous.

\subsection{OCT System and Data Acquisition}

An ultrahigh-speed spectral domain OCT system was used to perform en face Doppler OCT measurements of total retinal blood flow. The same system is described by Choi et al. ${ }^{28}$ and, therefore, is only briefly summarized. The system uses a commercially available superluminescent diode (Superlum, SLD-371) with a full-width-at-half-maximum bandwidth of 55-nm and an 840$\mathrm{nm}$ central wavelength. A line scan camera (Basler Sprint spL4096-140 km) with 832 illuminated pixels was used to achieve an imaging speed of 244,000 A-scans per second. The axial resolution and total imaging range, in tissue, were $5.7 \mu \mathrm{m}$ and $1.5 \mathrm{~mm}$, respectively. OCT power incident on the cornea was $2.5 \mathrm{~mW}$, and the system sensitivity was $99 \mathrm{~dB}$.

Total retinal blood flow was calculated using a technique described previously. ${ }^{28}$ In brief, a $200 \mu \mathrm{m} \times 200 \mu \mathrm{m}$ area centered at the optic nerve head was repeatedly scanned at a $83-\mathrm{Hz}$ volume acquisition rate with $150 \mathrm{~A}$-scans per B-scan, $25 \mathrm{~B}$ scans per volume (scan duty cycle of $0.85 \%$ ), and 100 repeated volumes per acquisition. Total retinal arterial blood flow was calculated by segmenting the central retinal artery and integrating the axial velocity in the en face plane, avoiding the need to measure Doppler angles. The volume acquisition rate used in

Table 1 Number and weight of rats by protocol.

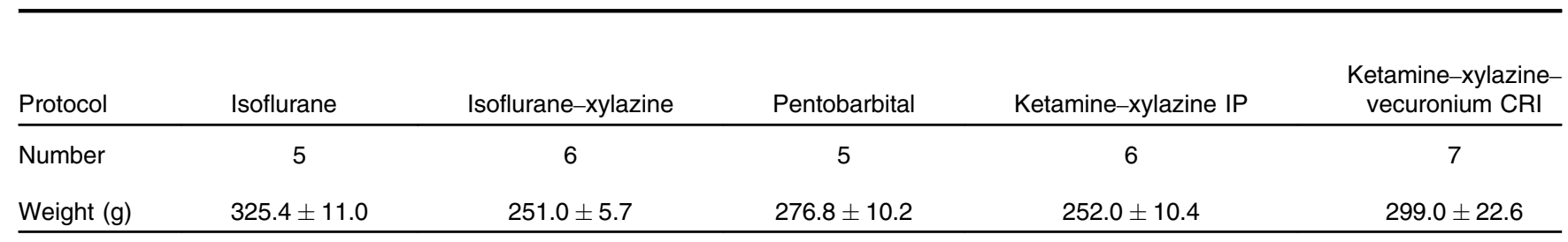

Note: CRI, continuous rate infusion; IP, intraperitoneal. 
this study was $>10 \times$ faster than typical heart rates encountered in rats under anesthesia, which enabled accurate measurement of mean total retinal arterial blood flow by averaging retinal arterial blood flow over multiple cardiac cycles.

\subsection{Subjective Evaluation of Anesthetic Protocols}

Each anesthetic protocol was subjectively evaluated according to the following criteria: (1) time-stability of blood flow, (2) overall rate of blood flow, (3) immobilization of the eye, and (4) simplicity. Criterion (1) is intended to capture whether a given anesthetic protocol has a reasonable window of time, say $20 \mathrm{~min}$, over which the blood flow values are reasonably constant, trending neither higher nor lower; the existence of such a time window is important for obtaining repeatable blood flow measurements. For criterion (2), while it would be ideal to discuss retinal blood flow rates relative to those seen in unanesthetized rats, to our knowledge such values have not been reported. Thus, for the purposes of this study, we describe the blood flow rate associated with a given anesthetic protocol relative to blood flow rates produced by the other tested anesthetic protocols: if a certain anesthetic protocol is associated with a higher blood flow rate than the others, we will refer to its blood flow values as "high." We use the converse definition for "low" blood flow rates. Criterion (3) is intended to capture whether a given anesthetic protocol facilitates eye imaging by minimizing ocular motion: as discussed in subsequent sections, it is our experience that with certain anesthetic protocols the rat eye tends to "drift," making it difficult to obtain good OCT beam alignment.

\section{Results}

Table 3 summarizes the measured physiological parameters, separated according to anesthetic protocol. Figure 1 shows, for each of the tested anesthetic protocols, total retinal blood flow, heart rate, and mean blood pressure measurements as function of time. For a given anesthetic protocol, different style markers correspond to different rats. Not all rats have the same frequency of blood flow measurements, which is generally a reflection of the difficulty of sufficiently stabilizing the eye for imaging. Furthermore, due to the anesthetic agents' half-lives, the time over which the measurements were obtained was shorter for the IP delivered protocols than for CRI and inhalation protocols. For some blood flow measurements, the blood pressure cuff failed to obtain measurements, and, consequently, not all blood flow measurements have associated blood pressure measurements. Furthermore, one rat imaged under ketaminexylazine IP did not have IOP measurements due to the tonometer malfunctioning. Table 4 summarizes the qualitative evaluation of the different anesthetic protocol with respect to the criteria described in Sec. 2.3.

\section{Discussion}

With reference to Fig. 1, the blood flow measurements for the isoflurane and isoflurane-xylazine anesthetic protocols were markedly higher than for the other three anesthetic protocols. This observation is consistent with MRI studies, as well as the fact that isoflurane is a known vasodilator. ${ }^{14,15}$ When using isoflurane alone, we found that eye motion made imaging challenging. The relative scarcity of measurements collected under isoflurane anesthesia is a reflection of this difficulty. While we found that IP injection of xylazine in conjunction with isoflurane significantly reduced eye motion, the vasodilatory effects of isoflurane may make it an unsuitable anesthetic for investigating neurovascular coupling where vessel dilation is coupled to the stimulus (as is the case with flicker light stimulus). Finally, note that neither isoflurane nor isoflurane-xylazine anesthesia showed strong time transients for blood flow, heart rate, or blood pressure.

Pentobarbital was found to yield reasonably time-invariant blood flows at rates lower than those observed when using isoflurane. However, heart rate and mean blood pressure under pentobarbital appear to show a relatively large variation in values, although no clear trend is observable. Finally, anesthesia using pentobarbital did not reduce eye motion, making imaging difficult. One potential solution to eye motion, not explored in this paper, is to combine pentobarbital with vecuronium.

The blood flow versus time plots measured with the ketamine-xylazine IP protocol exhibited a clear time-dependence, with blood flow and mean blood pressure decreasing with time and heart rate increasing with time. This time-dependence, presumably due to the IP bolus delivery, complicates the ability to acquire repeatable measurements among different animals. In a previous study examining ketamine-xylazine IP anesthesia (ketamine: $125 \mathrm{mg} / \mathrm{kg}$ and xylazine: $10 \mathrm{mg} / \mathrm{kg}$ ) for adult rats (8- to 12-weeks old; 350 to $400 \mathrm{~g}$ ), voluntary movement occurred $\sim 1.5 \mathrm{~h}$ after ketamine-xylazine injection and the half-life of both ketamine and xylazine was found to be $\sim 1.3 \mathrm{~h} .{ }^{36}$ The time transients observed in our data are consistent with these findings. Another interesting finding from that same study is that ketamine and xylazine clearance was strongly reduced in older rats (2.0- to 2.4-years old; 0.8 to $1.1 \mathrm{~kg}$ ): in old rats, the half-lives of ketamine and xylazine were found to be 8.5

Table 3 Summary of the measured physiological parameters.

\begin{tabular}{|c|c|c|c|c|c|}
\hline Anesthetic protocol & $\operatorname{TRBF}(\mu \mathrm{L} / \min )$ & $\mathrm{HR}(\mathrm{BPM})$ & $\mathrm{MBP}(\mathrm{mmHg})$ & IOP $(\mathrm{mmHg})$ & $\mathrm{SO}_{2}(\%)$ \\
\hline Isoflurane & $8.2 \pm 2.1$ & $358.5 \pm 36.8$ & $98.9 \pm 12.9$ & $10.8 \pm 1.3$ & $98.1 \pm 1.4$ \\
\hline Isoflurane-xylazine & $8.9 \pm 2.1$ & $326.5 \pm 22.3$ & $72.4 \pm 14.0$ & $9.4 \pm 0.8$ & $95.4 \pm 1.4$ \\
\hline Pentobarbital & $4.7 \pm 1.3$ & $422.8 \pm 30.9$ & $93.5 \pm 24.3$ & $10.1 \pm 1.0$ & $98.6 \pm 1.1$ \\
\hline Ketamine-xylazine IP & $3.7 \pm 1.4$ & $289.9 \pm 14.8$ & $93.9 \pm 14.3$ & $8.8 \pm 1.0$ & $98.5 \pm 0.8$ \\
\hline Ketamine-xylazine-vecuronium CRI & $3.1 \pm 1.0$ & $304.8 \pm 20.9$ & $76.2 \pm 11.8$ & $8.5 \pm 0.6$ & $97.0 \pm 1.6$ \\
\hline
\end{tabular}

Note: All values are listed as mean \pm standard deviation. TRBF, total retinal blood flow; HR, heart rate; BPM, beats per minute; MBP, mean blood pressure; IOP, intraocular pressure; $\mathrm{SO}_{2}$, oxygen saturation; IP, intraperitoneal; CRI, continuous rate infusion. 

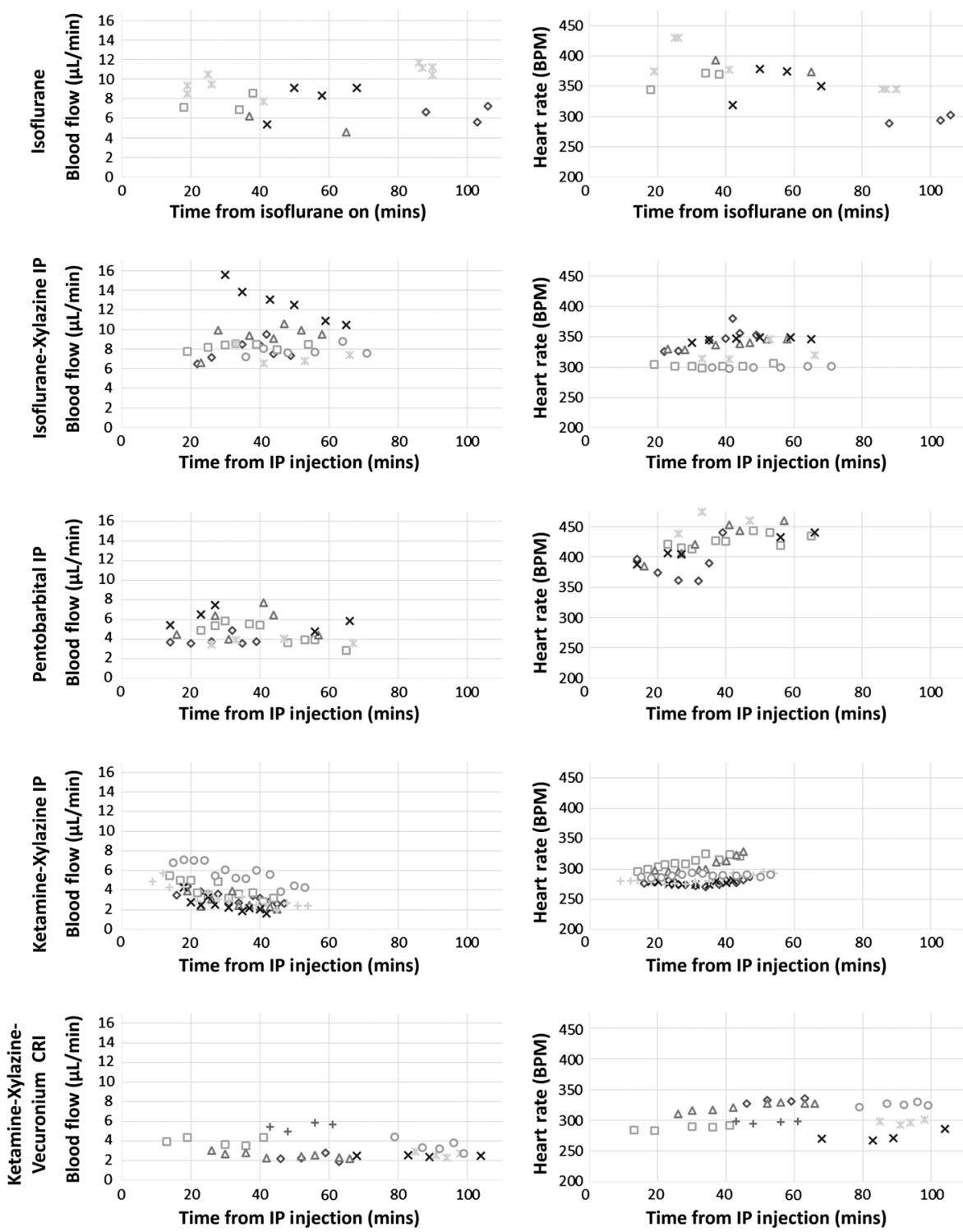
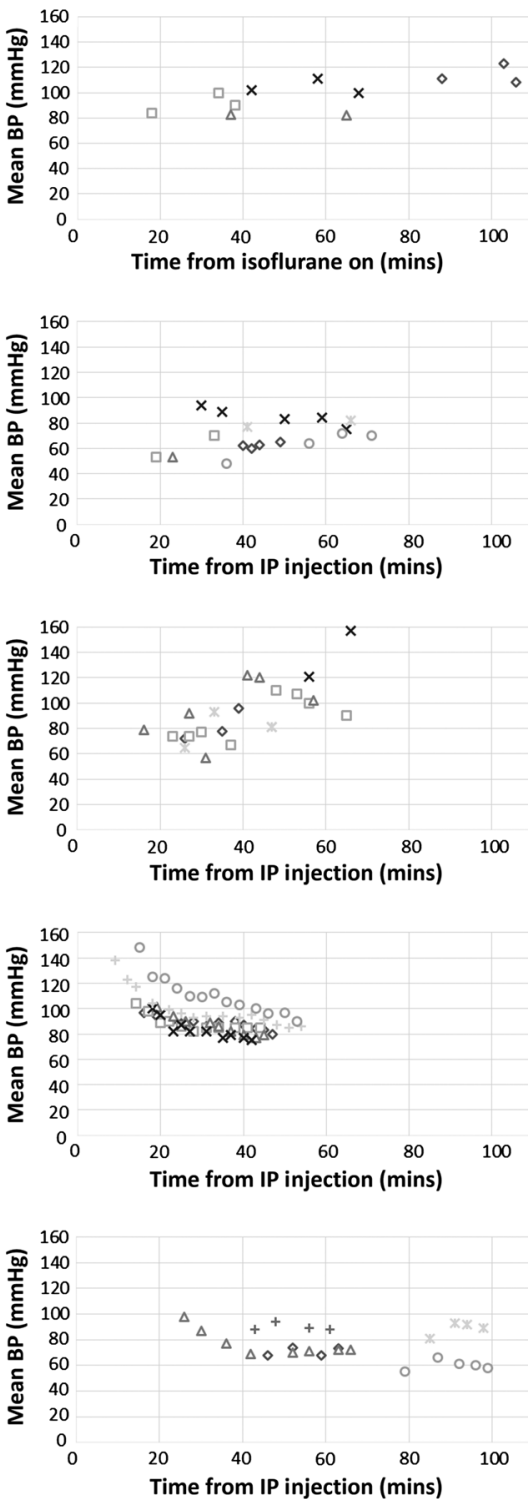

Fig. 1 Total retinal blood flow, heart rate, and mean blood pressure measurements as a function of time for the five tested anesthetic protocols. For a given anesthetic protocol, different style markers correspond to different rats. BPM, beats per minute; IP, intraperitoneal; CRI, continuous rate infusion.

Table 4 Qualitative assessment of anesthetic protocols.

\begin{tabular}{lcccc}
\hline & \multicolumn{2}{c}{ Time- } & Blood flow \\
Anesthetic protocol & stability & rate & $\begin{array}{c}\text { Ocular } \\
\text { immobilization }\end{array}$ & Simplicity \\
\hline Isoflurane & $\checkmark$ & High & $x$ & $\checkmark$ \\
Isoflurane-xylazine & $\checkmark$ & High & $\checkmark$ & $\checkmark$ \\
$\begin{array}{l}\text { Pentobarbital } \\
\text { Ketamine-xylazine IP }\end{array}$ & $x$ & Low & $x$ & $\checkmark$ \\
$\begin{array}{l}\text { Ketamine-xylazine- } \\
\text { vecuronium CRI }\end{array}$ & $\checkmark$ & Low & $\checkmark$ & $\checkmark$
\end{tabular}

Note: Check marks indicate that the anesthetic protocol was subjectively determined to have favorable characteristics with respect to the given criteria; cross marks indicate the converse. "High" and "low" blood flow values were subjectively assessed relative to the other anesthetic protocols, as described in Sec. 2.3. CRI, continuous rate infusion; IP, intraperitoneal. and $13 \mathrm{~h}$, respectively. This suggests that the time-dependencies observed in this study may change as a function of animal age.

Ketamine-xylazine-vecuronium anesthesia yielded low, relatively stable blood flows. CRI largely removed the time transients that were observed with ketamine-xylazine IP delivery, and heart rate and blood pressure were fairly constant in time. Although not included in this study, we have found that at the relatively low dose of ketamine-xylazine used in this anesthetic protocol (20 mg/kg/h ketamine and $2 \mathrm{mg} / \mathrm{kg} / \mathrm{hr}$ xylazine), eye movement was too severe to perform OCT imaging. Rather than increasing the dose of ketamine-xylazine (as we did in the case of the ketamine-xylazine IP protocol), we chose to minimize eye motion by adding vecuronium while maintaining the minimal dose of ketamine-xylazine for anesthesia; this choice was made so as to minimally perturb blood flow. A drawback of the ketamine-xylazine-vecuronium protocol is the relative complexity associated with delivering multiple drugs intravenously as well as the need to intubate and ventilate the animal due to paralysis. These challenges are somewhat increased in 
longitudinal studies, which involve nonterminal procedures, and therefore require that animals be carefully weaned off of the paralytic. We believe, however, that the stability and ease-of-imaging may justify the complexity.

There are a number of important limitations to our study. Certain physiological parameters, including $\mathrm{PO}_{2}, \mathrm{PCO}_{2}$, blood glucose, and $\mathrm{pH}$ were not measured or controlled, in large part because measurement of these parameters requires direct arterial cannulation, which complicates the procedure, particularly in longitudinal studies. Lacking knowledge of these parameters means that it is possible that at times we were imaging in the hyperoxic/hypoxic and/or hypercapniac/hypocapniac regimes. Other parameters, such as blood pressure, were measured using techniques known to be less accurate than invasive methods (e.g., we measured blood pressure using a blood pressure cuff instead of via direct arterial cannulation). Furthermore, in our study, only rats anesthetized with ketamine-xylazinevecuronium were ventilated, while ventilation could be used in conjunction with any of the tested anesthetic protocols. Because isoflurane is known to be a potent respiratory depressant, potentially inducing hypercapnia during spontaneous breathing, and because previous studies have shown that hypercapnia is associated with increased ocular blood flow due to vasodilation, the higher blood flows observed in rats anesthetized with isoflurane may be partially due to hypercapnia, which could be controlled in part by ventilation. While these limitations in monitoring and physiological control prevent us from ascribing the observed blood flow differences to the tested anesthetic agents, per se, they do not affect the goal of evaluating the effects of the different anesthetic protocols, taken as a whole. Moreover, a secondary aim of this study was to investigate anesthetic protocols commonly used in optical imaging of the small animal eye. This is especially true when our study is considered in the context of optical imaging studies of small animal retinal blood flow, where the above parameters are rarely measured or controlled. Another limitation of this study is that we did not test different drug dosages or consider all of the various methods of delivery. While such data would be informative, the number of animals needed to perform this analysis serves as a high barrier. Furthermore decreased dosages are likely to exacerbate ocular motion, making optical imaging difficult. Other limitations of the study include (1) that the protocols were evaluated using criteria that emphasized measurement precision rather than accuracy, which could lead to incorrect conclusions being reached when the results of this study are extended to disease models, and (2) that there was an absence of blood flow measurements from unanesthetized rats, which would have served as a baseline against with which the tested anesthetic protocols could be compared. Both of these limitations could be addressed in future studies by performing blood flow measurements using the microsphere method; this method has the distinct advantage that it can be performed in the absence of anesthesia. Despite these limitations, we believe that this study makes a useful contribution to the current functional blood flow imaging literature.

\section{Conclusion}

Measuring retinal blood flow in the small animal eye is important for studies of retinal disease pathogenesis and pharmaceutical development. However, anesthesia, which is a requirement for ocular imaging in small animals, may alter ocular blood flow. In this study, we used en face Doppler OCT to quantitatively investigate the influence of five different anesthetic protocols on total retinal blood flow in the rat. Additionally, each anesthetic protocol was qualitatively evaluated according to criteria relevant to functional blood flow imaging studies. We observed that different anesthetic protocols produced markedly different blood flows and that different anesthetic protocols varied with respect to the qualitative criteria. These findings suggest that the choice of anesthetic protocol should be carefully considered when designing and interpreting functional blood flow studies in the small animal eye.

\section{Disclosures}

Edward P. Feener is a cofounder and chief scientific officer of KalVista Pharmaceuticals Ltd.

\section{Acknowledgments}

This work was supported by grants from the National Institute of Health (NIH R01-EY011289-29, R44-EY022864, R01CA075289-16) and the Air Force Office of Scientific Research (FA9550-15-1-0473 and FA9550-12-1-0499).

\section{References}

1. G. Garhofer et al., "Use of the retinal vessel analyzer in ocular blood flow research," Acta Ophthalmol. 88(7), 717-722 (2010).

2. J. J. Weiter, R. A. Schachar, and J. T. Ernest, "Control of intraocular blood flow: I. intraocular pressure," Invest. Ophthalmol. Visual Sci. 12(5), 327 (1973).

3. L. Wang et al., "Microspheres method for ocular blood flow measurement in rats: size and dose optimization," Exp. Eye Res. 84(1), 108-117 (2007).

4. S. Orgul et al., "Measurement of optic nerve blood flow with nonradioactive colored microspheres in rabbits," Microvasc. Res. 51(2), 175-186 (1996).

5. C. Riva, B. Ross, and G. B. Benedek, "Laser Doppler measurements of blood flow in capillary tubes and retinal arteries," Invest. Ophthalmol. Visual Sci. 11(11), 936-934 (1972).

6. T. Tanaka, C. Riva, and I. Ben-Sira, "Blood velocity measurements in human retinal vessels," Science 186(4166), 830-831 (1974).

7. C. Pournaras and C. Riva, "Retinal blood flow evaluation," Ophthalmologica 229, 61-74 (2013).

8. C. Riva et al., "Blood velocity and volumetric flow rate in human retinal vessels," Invest. Ophthalmol. Visual Sci. 26(8), 1124 (1985).

9. R. Bonner and R. Nossal, "Model for laser Doppler measurements of blood flow in tissue," Appl. Opt. 20, 2097-2107 (1981).

10. C. E. Riva et al., "Laser Doppler flowmetry in the optic nerve," Exp. Eye Res. 55(3), 499-506 (1992).

11. J. D. Briers and A. F. Fercher, "Retinal blood-flow visualization by means of laser speckle photography," Invest. Ophthalmol. Visual Sci. 22(2), 255 (1982).

12. A. F. Fercher and J. D. Briers, "Flow visualization by means of singleexposure speckle photography," Opt. Commun. 37(5), 326-330 (1981).

13. D. A. Boas and A. K. Dunn, "Laser speckle contrast imaging in biomedical optics," J. Biomed. Opt. 15(1), 011109 (2010).

14. E. R. Muir and T. Q. Duong, "MRI of retinal and choroidal blood flow with laminar resolution," NMR Biomed. 24(2), 216-223 (2011).

15. Y. Li, H. Cheng, and T. Q. Duong, "Blood-flow magnetic resonance imaging of the retina," Neurolmage 39(4), 1744-1751 (2011).

16. Q. Peng et al., "MRI of blood flow of the human retina," Mag. Reson. Med. 65, 1768-1775 (2011).

17. W. E. Lieb et al., "Color Doppler imaging of the eye and orbit: technique and normal vascular anatomy," Arch. Ophthalmol. 109, 527-531 (2011).

18. Z. Butt et al., "Color Doppler imaging in untreated high- and normalpressure open-angle glaucoma," Invest. Ophthalmol. Visual Sci. 38, 690-696 (1997).

19. T. A. Ciulla et al., "Color Doppler imaging discloses reduced ocular blood flow velocities in nonexudative age-related macular degeneration," Am. J. Ophthalmol. 128, 75-80 (1999). 
20. B. R. White et al., "In vivo dynamic human retinal blood flow imaging using ultra-high-speed spectral domain optical coherence tomography," Opt. Express 11, 3490-3497 (2003).

21. J. A. Izatt et al., "In vivo bidirectional color Doppler flow imaging of picoliter blood volumes using optical coherence tomography," Opt. Lett. 22, 1439-1441 (1997).

22. R. A. Leitgeb et al., "Real-time measurement of in vitro flow by Fourierdomain color Doppler optical coherence tomography," Opt. Lett. 29, 171-173 (2004).

23. Y. Wang et al., "In vivo total retinal blood flow measurement by Fourier domain Doppler optical coherence tomography," J. Biomed. Opt. 12, 041215 (2007).

24. S. Makita, T. Fabritius, and Y. Yasuno, "Quantitative retinal-blood flow measurement with three-dimensional vessel geometry determination using ultrahigh-resolution Doppler optical coherence angiography," Opt. Lett. 33, 836-838 (2008).

25. Y. Wang et al., "Measurement of total blood flow in the normal human retina using Doppler Fourier-domain optical coherence tomography," Br. J. Ophthalmol. 93, 634-637 (2009).

26. B. Baumann et al., "Total retinal blood flow measurement with ultrahigh speed swept source/Fourier domain OCT," Biomed. Opt. Express 2, 1539-1552 (2011).

27. T. Schmoll, C. Kolbitsch, and R. A. Leitgeb, "Ultra-high-speed volumetric tomography of human retinal blood flow," Opt. Express 17, 4166-4176 (2009).

28. W. Choi et al., "Measurement of pulsatile total blood flow in the human and rat retina with ultrahigh speed spectral/Fourier domain OCT," Biomed. Opt. Express 3, 1047-1061 (2012).
29. Y. Wang et al., "Pilot study of optical coherence tomography measurement of retinal blood flow in retinal and optic nerve diseases," Invest. Ophthalmol. Visual Sci. 52, 840-845 (2011).

30. Z. Zhi et al., "Volumetric and quantitative imaging of retinal blood flow in rats with optical microangiography," Biomed. Opt. Express 2, 579-591 (2011).

31. Z. Zhi et al., "Optical microangiography of retina and choroid and measurement of total retinal bloodflow in mice," Biomed. Opt. Express 3, 2976-2986 (2012).

32. V. J. Srinivasan et al., "Quantitative cerebral blood flow with optical coherence tomography," Biomed. Opt. Express 18, 2477-2494 (2010).

33. K. S. Hendrich et al., "Cerebral perfusion during anesthesia with fentanyl, isoflurane, or pentobarbital in normal rats studied by arterial spinlabeled MRI," Magn. Reson. Med. 46, 202-206. (2001).

34. K. K. K. J. W. Langsjo et al., "Effects of subanesthetic doses of ketamine on regional cerebral blood flow, oxygen consumption, and blood volume in humans," Anesthesiology 99, 614-623 (2003).

35. K. Sicard et al., "Regional cerebral blood flow and BOLD responses in conscious and anesthetized rats under basal and hypercapnic conditions: implications for functional MRI studies," J. Cerebral Blood Flow Metab. 23, 472-481 (2003).

36. D. Veilleux-Lemieux et al., "Pharmacokinetics of ketamine and xylazine in young and old Sprague-Dawley rats," J. Am. Assoc. Lab. Anim. Sci. 52, 567-570 (2013).

Biographies for the authors are not available. 